\title{
Improved Outcomes for Prostate Cancer Using Hypofractionated Radiotherapy and Dose Escalation to 55Gy
}

Joanna Mackenzie ${ }^{1}$, Alastair Law ${ }^{1^{*}}$, Jahangeer Malik ${ }^{1}$, Gill Kerr ${ }^{1}$, Grahame Howard ${ }^{1}$, Geoff Higgins ${ }^{1,2}$ and Duncan Mclaren $^{1}$

${ }^{1}$ Department of Clinical Oncology, Edinburgh Cancer Centre, Western General Hospital, Edinburgh, UK

${ }^{2}$ Gray Institute for Radiation Oncology and Biology, Old Road Campus Research Building, Oxford University, Oxford, UK

"Corresponding author: Alastair Law, Department of Clinical Oncology, Edinburgh Cancer Centre, Western General Hospital, Edinburgh, UK, Tel: +441315372211; Email: alastair.law@luht.scot.nhs.uk

Received date: Jun 17, 2014, Accepted date: Aug 12, 2014, Publication date: Aug 31, 2014

Copyright: () 2014 Mackenzie J, et al. This is an open-access article distributed under the terms of the Creative Commons Attribution License, which permits unrestricted use, distribution, and reproduction in any medium, provided the original author and source are credited.

\begin{abstract}
Introduction: This Centre has previously reported outcomes for patients treated with neoadjuvant hormone therapy (NAHT) and 52.5Gy in 20 fractions. We now present the outcome data for patients treated with 55Gy in 20 fractions and NAHT.

Methods and Materials: 584 patients were treated for T1-T3 node negative prostate cancer. The median age was 67.2 years (range 49-80). All patients received 3 months of NAHT followed by CT planned conformal radiotherapy to the prostate using $55 \mathrm{~Gy}$ in 20 fractions. 147 patients considered at particularly high risk of relapse also received 2 years of adjuvant androgen deprivation. Outcomes were obtained through serial PSA measurement. Patients were classified into prognostic groups according to Zelefsky criteria. PSA relapse was defined according to Houston criteria. The outcomes with 55Gy were compared with the 52.5Gy cohort.
\end{abstract}

Results: The 10 year cause specific survival increased from $67.3 \%$ with $52.5 \mathrm{~Gy}$ to $92.9 \%$ in the $55 \mathrm{~Gy}$ group. Patients receiving $55 \mathrm{~Gy}$ had better outcomes than those receiving $52.5 \mathrm{~Gy}$ in all prognostic groups with or without adjuvant hormone use. PSA relapse free survival at five years in the patients who did not receive adjuvant hormones are:- 80.2 v $63.6 \%$ in the 'Good', 69.5 v $43.7 \%$ in the 'Intermediate' and 40.3 v $15.3 \%$ in the 'Poor' prognostic groups $(p<0.01)$.

Conclusions: Our results demonstrate improved outcomes with prostate radiotherapy across all prognostic groups with a modest dose escalation from 52.5 to $55 \mathrm{~Gy}$ in 20 fractions. This supports evidence of a steep dose response gradient and a low alpha beta ratio in this cancer.

Keywords: Prostate cancer; Hypofractionated radiotherapy; Disease free survival; Overall survival

\section{Introduction}

External beam radiotherapy in the treatment of localised prostate cancer has evolved significantly in recent years. The changes in therapy have been in three main areas: the addition of systemic hormone therapy in the neoadjuvant and adjuvant settings, improvements in treatment delivery with more conformal radiotherapy, intensity modulated radiotherapy (IMRT) and imageguided radiotherapy (IGRT) and in dose escalation both in standard 1.8-2 Gy fractions and using 2.75-3Gy hypofractionation. There are ongoing studies of dose escalation in this setting and the optimal dose for radical local therapy is as yet undetermined.

Previously Edinburgh Cancer Centre has published outcomes from a series of 300 patients treated with 52.5 Gy in 20 fractions of radical radiotherapy to the prostate [1]. We now present a retrospective case series of 584 patients treated with a modest dose escalation to $55 \mathrm{~Gy}$ in 20 fractions over 4 weeks, including a comparison with the $52.5 \mathrm{~Gy}$ data.

Neoadjuvant androgen therapy (NAHT) is now standard practice in combination with external beam radiotherapy to the prostate for patients with intermediate or high risk disease. Using 3 to 6 months of NAHT reduces the risk of PSA and local relapse [2].The addition of adjuvant hormone therapy (AHT) using LHRH agonists has a significant impact both on the risk of local relapse and on overall survival. These improvements are seen predominantly in those patients with high risk disease (Gleason 7-10) [3].

All patients in our 52.5 and 55 Gy series were treated with NAHT and those considered high risk in our 55 Gy series also had adjuvant hormone therapy for 2 years.

\section{Materials and Methods}

\section{Treatment}

Patients were identified through review of the regional oncology database, and were treated between 2001 and 2005. 584 men with T1-3 N0M0 and histologically confirmed prostate cancer received NAHT using LHRH agonist for 3 to 6 months prior to radical radiotherapy following MDT discussion of each case.

Radiotherapy was planned using 3D conformal CT planning with CTV defined as the whole prostate gland \pm base of seminal vesicles depending on the calculated risk of seminal vesicle involvement based on the Roach formula - PSA + ((GS-6) x10) [4]. The CTV included the 
Citation: Mackenzie J, Law A, Malik J, Kerr G, Howard G, et al. (2014) Improved Outcomes for Prostate Cancer Using Hypofractionated

Page 2 of 5

proximal $2 \mathrm{~cm}$ of the seminal vesicles if they were calculated as $\geq 15 \%$ risk of seminal vesicle involvement or this had been demonstrated on staging MRI scan. Pelvic nodes were not treated. The CTV to PTV margin was $1 \mathrm{~cm}$ supero-inferiorly and anterolaterally and $0.7 \mathrm{~cm}$ posteriorly. Patients were treated with 55 Gy in 20 daily fractions over 4 weeks prescribed to isocentre.

AHT for 2 years was offered to patients with Gleason Score $\geq 8$ or other high risk features such as a Gleason 7 cancer with PSA over 20 or T3 disease.

\section{Follow up}

Follow up with serial PSA measurement was 3 monthly in the first year following treatment than 6 monthly thereafter. This took place initially in secondary care and subsequently with the GP and follow up via postal system. The median follow-up was 81 months. Three patients were lost to follow up at 39, 44 and 58 months, otherwise the minimum follow up was 6 years.

\section{Outcomes}

The primary outcome measure was PSA relapse free survival. Secondary outcomes were overall survival and cause specific survival. Details of follow up PSA measurements and restarting of hormone therapy on relapse were obtained through case note review, interrogation of the laboratory result system or direct contact with the patient's general practitioner. The data for the 52.5 Gy cohort was updated from our previous paper. PSA relapse is defined by Houston criteria [5] as a rise of $>2 \mathrm{ng} / \mathrm{ml}$ above the nadir (lowest recorded value). Any patient with uncontrolled PSA at the time of death was considered to have prostate cancer present at time of death for the purposes of actuarial cause specific survival. Outcomes in this 55Gy cohort were compared with our previous series of men who received $52.5 \mathrm{~Gy}$.

\section{Statistical analysis}

Statistical analysis was undertaken using SAS software. Survival and relapse were measured from the date of starting NAHT. Actuarial relapse and survival rates were estimated using the Kaplan-Meier technique and compared using the log-rank test. A multivariate analysis for factors affecting outcome was performed using the proportional hazards model. Pre-treatment PSA, Gleason score and tumour stage are recognized as prognostic factors. Patients were grouped into good (PSA $\leq 10$, Gleason score $\leq 6$, and Stage T1/T2), intermediate (1 raised value), and poor ( 2 or more raised values) prognostic groups) using recognised standard criteria originally described by Zelefsky et al. [6] Prognostic groupings were determined on the basis of pre-treatment staging.

\section{Results}

The baseline characteristics of all 584 men receiving 55Gy and the 301 men who received 52.5Gy are demonstrated in Table 1. All patients received NAHT. In addition, 147 patients in the 55Gy group received 2 years of AHT. These patient populations are considered separately.

\begin{tabular}{|c|c|c|c|}
\hline Dose & \multicolumn{2}{|l|}{$55 \mathrm{~Gy}$} & $52.5 \mathrm{~Gy}$ \\
\hline \multirow[t]{2}{*}{ Adjuvant Hormones } & $2 \mathrm{yrs}$ & None & None \\
\hline & \multicolumn{2}{|l|}{584} & \\
\hline Total & 147 & 437 & 301 \\
\hline Years & \multicolumn{2}{|l|}{$2001-2005$} & $1995-2001$ \\
\hline \multicolumn{4}{|l|}{ Age } \\
\hline Mean & 66.6 & 67.4 & 67.7 \\
\hline Median & 68 & 68 & 69 \\
\hline Range & $51-78$ & $49-80$ & $47-80$ \\
\hline \multicolumn{4}{|l|}{ Stage (NO MO) } \\
\hline $\mathrm{T} 1$ & $21(14.3 \%)$ & $130(29.8 \%)$ & $53(17.7 \%)$ \\
\hline $\mathrm{T} 2$ & $64(43.5 \%)$ & $244(55.8 \%)$ & 149 (49.3\%) \\
\hline T3 & $62(42.2 \%)$ & $63(14.4 \%)$ & $99(33.0 \%)$ \\
\hline \multicolumn{4}{|l|}{ Gleason } \\
\hline 4 & 0 & $3(0.7 \%)$ & $27(9.0 \%)$ \\
\hline 5 & 0 & $14(3.2 \%)$ & $43(14.3 \%)$ \\
\hline 6 & $3(2.0 \%)$ & $195(44.6 \%)$ & $88(29.3 \%)$ \\
\hline 7 & $47(32.0 \%)$ & $210(48.1 \%)$ & $88(29.3 \%)$ \\
\hline 8 & $56(38.1 \%)$ & $10(2.3 \%)$ & $37(12.3 \%)$ \\
\hline 9 & $39(26.5 \%)$ & $5(1.1 \%)$ & $16(5.3 \%)$ \\
\hline 10 & $2(1.4 \%)$ & 0 & $20.7 \%)$ \\
\hline \multicolumn{4}{|l|}{ Presenting PSA } \\
\hline$<=10$ & $29(19.7 \%)$ & $165(37.8 \%)$ & $68(22.7 \%)$ \\
\hline$>10<=20$ & $34(23.1 \%)$ & $165(37.8 \%)$ & $100(33.3 \%)$ \\
\hline$>20<=50$ & $60(40.8 \%)$ & $102(23.3 \%)$ & $96(32.0 \%)$ \\
\hline$>50$ & $24(16.3 \%)$ & $5(1.1 \%)$ & $36(12.0 \%)$ \\
\hline NK & & & 1 \\
\hline Median & 24.8 & 12.0 & 17.9 \\
\hline \multicolumn{4}{|l|}{ Surgery } \\
\hline TURP & $11(7.5 \%)$ & $29(6.6 \%)$ & $75(25.0 \%)$ \\
\hline Biopsy only & 136 & 408 & 226 \\
\hline \multicolumn{4}{|l|}{ Prognostic group } \\
\hline Good & 0 & $78(17.8 \%)$ & $37(12.3 \%)$ \\
\hline Intermediate & $22(15.0 \%)$ & $189(43.3 \%)$ & $103(34.3 \%)$ \\
\hline Poor & $125(85.0 \%)$ & $170(38.9)$ & $160(53.3 \%)$ \\
\hline NK & & & 12 \\
\hline
\end{tabular}

Table 1: Baseline patient characteristics. 
Citation: Mackenzie J, Law A, Malik J, Kerr G, Howard G, et al. (2014) Improved Outcomes for Prostate Cancer Using Hypofractionated

Within the 55 Gy cohort, no patients in the Zelefsky 'Good' prognostic group received AHT. All those patients in the 'Intermediate' group receiving AHT had a Gleason score of 7 or above with the exception of one patient who had a presenting PSA of $>50$. In the 'Poor' prognostic group receiving AHT, patients were more likely to be T3 $(\mathrm{p}<0.05)$, have a higher Gleason score $(\mathrm{p}<0.0001)$ and higher PSA at presentation $(\mathrm{p}<0.0001) .11$ patients with a Gleason score of 8 or 9 and 4 patients with PSA of 50+ did not receive AHT due to patient preference or comorbidities.

\section{PSA relapse}

The primary outcome measure was PSA relapse free survival. At 5 years, the overall PSA relapse rate was $3.4 \%$ in the 'Good' prognostic group, $19.4 \%$ in the 'Intermediate' prognostic group and $33.7 \%$ in the 'Poor' prognostic group. Figure 1 demonstrates the PSA relapse free survival by each prognostic group comparing those patients receiving 52.5 Gy and no AHT, 55 Gy with no AHT and 55 Gy with AHT.

Comparing the 52.5 Gy group and the 55 Gy group with and without AHT the differences in PSA relapse free survival at 5 years were statistically significant in all prognostic groups.

A total of 191 patients had a PSA relapse. The 10 year PSA relapse rates show there were ongoing PSA relapses between 5 and 10 years. The 10 year PSA relapse free survival rates are also demonstrated in Figure 1.

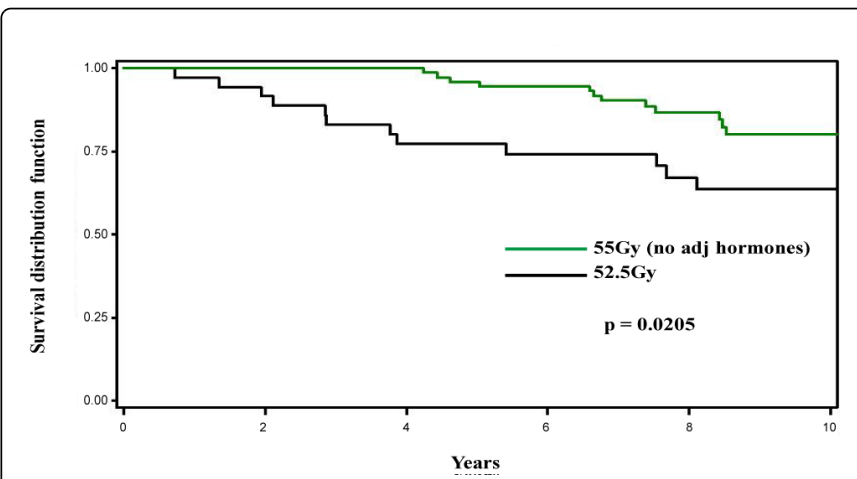

Figure 1 a): PSA relapse free survival rates for 'Good' prognostic groups.

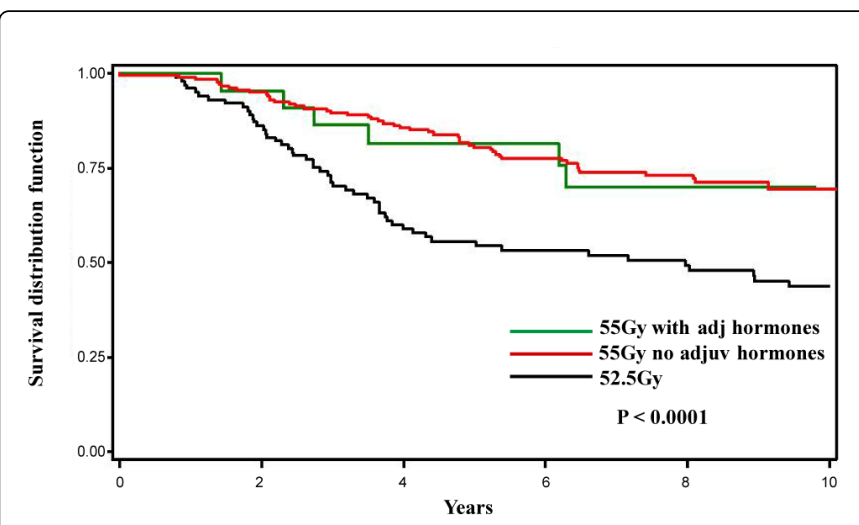

Figure 1 b): PSA relapse free survival rates for 'Intermediate' prognostic group.

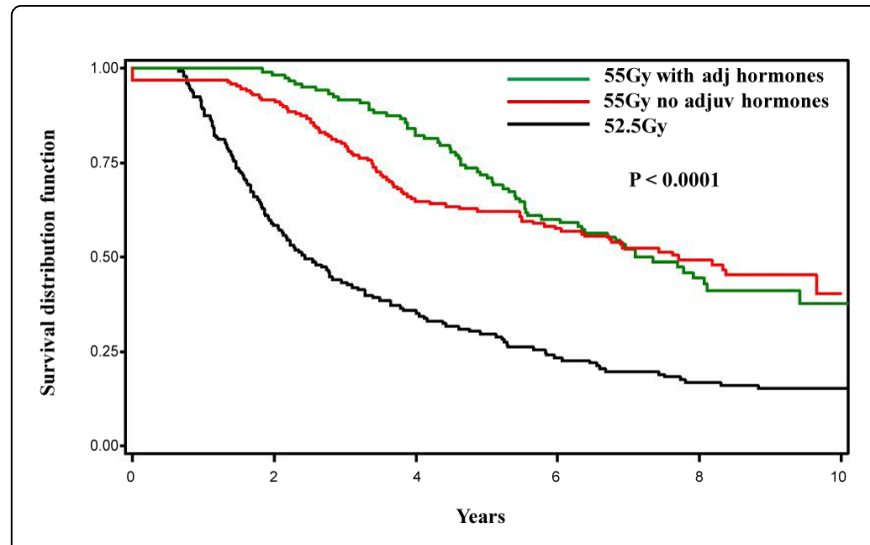

Figure 1c): PSA relapse free survival rates for 'Poor' prognostic group.

Multivariant analysis using proportional hazards ratio showed that the following variables were independent prognostic factors for PSA relapse in the 55Gy group: $\mathrm{T}$ stage $(\mathrm{P}<0.0001)$, Gleason score $(\mathrm{P}=0.0010)$ and age (older patients doing better $)(\mathrm{P}=0.0043)$. AHT and presenting PSA in this study were not significant.

\section{Survival}

The overall and cause specific survival rates (CSSR) at 5 and 10 years are presented in Table 2. The cause specific survival curves by treatment group are shown in Figure 2.

\begin{tabular}{|c|c|c|c|c|}
\hline \multirow{2}{*}{$\begin{array}{l}\text { Dose } \\
\text { Adjuvant hormones }\end{array}$} & \multicolumn{3}{|l|}{ 55Gy } & \multirow{2}{*}{$\begin{array}{l}52.5 \mathrm{~Gy} \\
\text { None }\end{array}$} \\
\hline & 2 years & None & Total & \\
\hline \multicolumn{5}{|l|}{ Survival @ 5yrs (\%) } \\
\hline Overall & 86.3 & 90.6 & 89.5 & 78.3 \\
\hline $\begin{array}{l}\text { Cause specific } \\
\text { survival rate (CSSR) }\end{array}$ & 93.4 & 97.8 & 96.7 & 84.7 \\
\hline \multicolumn{5}{|l|}{ By prognostic group } \\
\hline Good & N/A & 100 & 100 & 97.1 \\
\hline Intermediate & 90.5 & 97.8 & 97.0 & 92.9 \\
\hline Poor & 93.9 & 96.9 & 95.6 & 76.7 \\
\hline \multicolumn{5}{|l|}{$\begin{array}{l}\text { Survival @ 10yrs } \\
(\%)\end{array}$} \\
\hline Overall & 69.2 & 72.4 & 71.5 & 55.4 \\
\hline $\begin{array}{l}\text { Cause specific } \\
\text { survival rate (CSSR) }\end{array}$ & 82.3 & 92.9 & 90.2 & 67.3 \\
\hline \multicolumn{5}{|l|}{ By prognostic group } \\
\hline Good & N/A & 100.0 & 100.0 & 84.0 \\
\hline Intermediate & 85.7 & 95.3 & 94.3 & 83.2 \\
\hline Poor & 81.6 & 86.7 & 84.3 & 46.8 \\
\hline
\end{tabular}

Table 2: Overall survival and Cause specific survival rates at 5 and 10 years. 


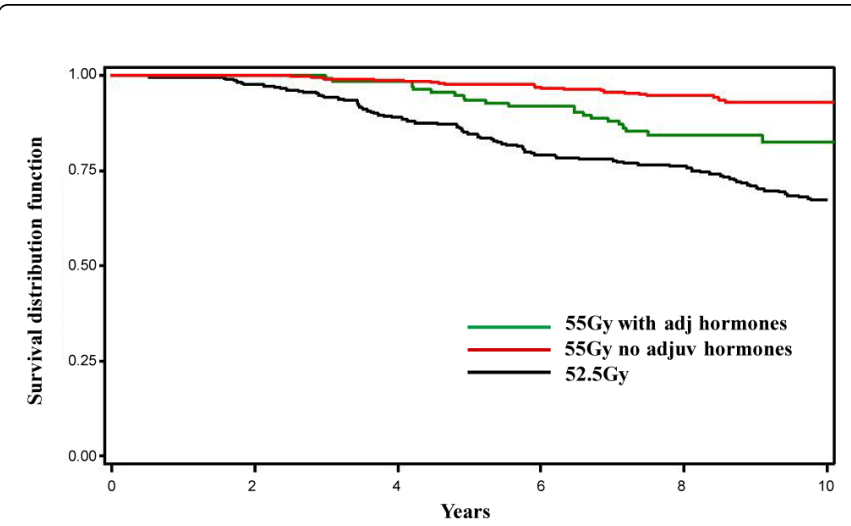

Figure 2: Cause specific survival rate curves.

Multivariant analysis using proportional hazards ratio showed that the only independent prognostic factor for cancer specific survival in the 55Gy group was Gleason score $(\mathrm{p}<0.0001)$.

\section{Discussion}

The reported outcomes in PSA relapse, cause specific and overall survival show statistical and clinically meaningful improvements in all outcomes with a modest dose escalation from 52.5Gy to 55Gy across all prognostic groups with or without the use of AHT. The overall 10 year PSA relapse rate in all patients groups (with or without AHT) receiving 55 Gy was $19.8 \%, 30.6 \%$ and $61.0 \%$ in the 'Good', 'Intermediate' and 'Poor' prognostic groups respectively. This has improved from $36.4 \%, 56.3 \%$ and $84.7 \%$, respectively in the $52.5 \mathrm{~Gy}$ cohort. The 10 year overall survival for all patients receiving $55 \mathrm{~Gy}$ was $71.5 \%$ and the cause specific survival rate (CSSR) was $90.2 \%$. If the patients who received AHT are excluded, then there are still significant improvements in PSA relapse rates, cause specific survival and overall survival.

The improvement with only a small dose increase is consistent with a low alpha beta ratio of prostate cancer cells and high dosefractionation sensitivity. Pooled data from seven studies of hypofractionation including 5969 patients determined an overall $\alpha / \beta$ value of 1.4 [7]. The dose of $55 \mathrm{~Gy}$ in these patients has approximate equivalence to $67 \mathrm{~Gy}$ in $2 \mathrm{~Gy}$ fractions using an $\alpha / \beta$ value of $1.4 \mathrm{~Gy}$ [7]. This would be considered a modest dose in comparison with current standard dose escalated regimens but our results are comparable or better than those of the recently reported comparative analysis by the PCRSG for external beam radiotherapy, which included studies using a minimum of $72 \mathrm{~Gy}$ [8]. We saw improvements in outcome after dose escalation from 52.5 to $55 \mathrm{~Gy}$ across low, intermediate and high risk prognostic groups which is in keeping with all these groups having a low $\alpha / \beta$ ratio and high dose fractionation sensitivity. This is supported by the meta-analysis from Mirabelle7 which found no significant differences in $\alpha / \beta$ ratio between prognostic groups.

Our results are consistent with another study that showed improvement in results with a small increase in dose when using hypofractionated regimes comparing 57 Gy in 19 fractions and 60 Gy in 20 fractions, delivered with intensity modulated radiotherapy (IMRT) in high risk patients only [9]. The 5 year biochemical progression free survival was 50\% with 57 Gy and 58\% with 60 Gy $(\mathrm{p}=0.45)$. The two regimens from the later study subsequently formed the exploratory arms of the CHiPP trial [10] which has not yet reported survival outcomes. Within our centre, the dose-escalated hypofractionated regime of $57 \mathrm{~Gy}$ in 19 fractions has been adopted since participating in the CHiPP study.

These improvements are substantial given the relatively small increase in dose and some of the improvement is likely to be due to other factors. This includes stage migration due to upgrading of cancers that would have previously been called Gleason 6 to Gleason 7 the Will Rogers Phenomenon [11]. Also the higher use of MRI scanning rather than CT scanning may have resulted in upstaging for example more patients may have evidence of $\mathrm{T} 3$ disease or greater pick up of bone or nodal metastases whilst previously they would have been offered radical radiotherapy. Also the greater awareness of prostate cancer may result in earlier presentation of men with less bulky disease or lower incidence of micrometastases within each of the prognostic groups.

The improvements in outcomes within this centre may also reflect changes in radiotherapy planning techniques with more conformal treatment plans and improved accuracy of dose delivery. It is noted however, that treatment delivery for all patients (in 52.5Gy and 55Gy) studied was prior to the use of fiducial seeds for IGRT and was based on matching bony anatomy only. Treatment was delivered using conventional conformal fields and none of the patients were treated using IMRT techniques.

In the group receiving 55 Gy some men with poor prognostic features received 2 year AHT. This is based on data that suggests a 5-8\% improvement in overall survival subgroups with AHT in higher grade prostate cancer [12]. A small part of the improvement in results in the 55Gy group may be partly due to this as none of the 52.5Gy received AHT. Those patients in the poor risk group who received AHT initially achieved better outcomes than those in the same groups who had no AHT with the PSA relapse free survival curves running parallel up to 5 years. However, it is noted that after 5 years the curves cross over and at 10 years the PSA relapse free survival as well as the overall survival is higher in the group who did not receive AHT. The reason for this is probably that the group who were assigned to AHT had worse baseline characteristics even if they were in the same prognostic group. The poor risk patient group who received AHT were statistically significantly more likely to have T3 disease, a higher median presenting PSA and to have a higher Gleason score than the poor risk patients who did not have AHT. Randomised studies have shown a sustained benefit in higher grade disease, but it may have been that 3 years or even lifelong of AHT may have had greater benefit than the two years we used perhaps in complete eradication of micrometastases rather than just delaying recurrence [13].

One of the limitations of our patient cohort is the lack of comprehensive acute and late toxicity data in both the 52.5 and 55Gy groups. Our database is able to reliably access PSA results on patients throughout the region but the toxicity data is not as comprehensive as patients may have been assessed or treated for radiation toxicity in district general hospitals and we may not always have received full documentation. Our recorded toxicities are however low and similar between the 52.5 and 55Gy groups (not reported in this paper) and similar to the recent $\mathrm{CHHiP}$ study that has published a preliminary safety analysis of conventional versus hypofractionated regimens [10]. In this study patients were randomised to 3 arms and were treated with standard $2 \mathrm{~Gy}$ fractions up to $74 \mathrm{~Gy}$ or, with $3 \mathrm{~Gy}$ fractions to a total of either 57 or $60 \mathrm{~Gy}$, respectively using IMRT. At a median FU of 50.5 months there were no differences in the incidence of Grade 2 
Citation: Mackenzie J, Law A, Malik J, Kerr G, Howard G, et al. (2014) Improved Outcomes for Prostate Cancer Using Hypofractionated Radiotherapy and Dose Escalation to 55Gy. J Nucl Med Radiat Ther 5: 188. doi:10.4172/2155-9619.1000188

Page 5 of 5

or worse RTOG bowel and bladder toxicities or reported sexual dysfunction scores across the 3 arms. This is reassuring data given the dose per fraction and total dose were both higher than our 55Gy cohort. Whilst in our centre we now use higher doses than 55 Gy we expect the recent introduction of IMRT to counterbalance any increase in toxicity.

The rationale for giving radiotherapy for prostate cancer has been confirmed by the SPCG-7/SFUO 3 trial [13] which investigated the addition of prostate radiotherapy to hormones in locally advanced or high risk disease. Patients were randomised to receive lifelong hormones \pm at least 70 Gy external beam radiotherapy to the prostate and seminal vesicles. The addition of radiotherapy improved the 10 year prostate cancer specific mortality from $23.9 \%$ to $11.9 \%$. In both this study and our study, radiotherapy was given to the prostate only despite many patients being considered at high risk of pelvic nodal involvement according to the Roach formula. Whether radiotherapy should also be given to the pelvic nodes is also a subject of debate. The significant improvements in our results by increasing the dose of radiotherapy to the prostate only across all risk groups is consistent with either there being a lower risk of nodal metastases than historical studies or that the use of hormones treatment eradicates microscopic disease. The PIVOTAL study is looking at the option of pelvic nodal irradiation using IMRT and it may be that a higher dose to the pelvic nodes in selected patients with high risk of nodal metastases may improve outcomes.

\section{Conclusions}

Within our centre we have demonstrated a significant and clinically important improvement in PSA relapse free survival, cause specific survival and overall survival for early prostate cancer with a modest dose escalation from 52.5 to $55 \mathrm{~Gy}$ using hypofractionated external beam radiotherapy. We have now increased our hypofractionated regime to $57 \mathrm{~Gy}$ in 19 fractions. Results of this, and also randomised studies comparing standard 2 Gy fractionations versus hypofractionated fractions, are awaited.

\section{References}

1. Higgins GS, McLaren DB, Kerr GR, Elliott T, Howard GC (2006) Outcome analysis of 300 prostate cancer patients treated with neoadjuvant androgen deprivation and hypofractionated radiotherapy. Int J Radiat Oncol Biol Phys 65: 982-989.

2. Denham JW, Steigler A, Lamb DS, Joseph D, Turner S, et al. (2011) Short-term neoadjuvant androgen deprivation and radiotherapy for locally advanced prostate cancer: 10-year data from the TROG 96.01 randomised trial. Lancet Oncol 12: 451-459.

3. Pilepich MV, Winter K, Lawton CA, Krisch RE, Wolkov HB, et al. (2005) Androgen suppression adjuvant to definitive radiotherapy in prostate carcinoma-long-term results of phase III RTOG 85-31. Int J Radiat Oncol Biol Phys 61: 1285-1290.

4. Diaz A, Roach M 3rd, Marquez C, Coleman L, Pickett B, et al. (1994) Indications for and the significance of seminal vesicle irradiation during 3D conformal radiotherapy for localized prostate cancer. Int J Radiat Oncol Biol Phys 30: 323-329.

5. Pickles T, Kim-Sing C, Morris WJ, Tyldesley S, Paltiel C (2003) Evaluation of the Houston biochemical relapse definition in men treated with prolonged neoadjuvant and adjuvant androgen ablation and assessment of follow-up lead-time bias. Int J Radiat Oncol Biol Phys 57: 11-18.

6. Zelefsky MJ, Leibel SA, Gaudin PB, Kutcher GJ, Fleshner NE, et al. (1998) Dose escalation with three-dimensional conformal radiation therapy affects the outcome in prostate cancer. Int J Radiat Oncol Biol Phys 41: 491-500.

7. Miralbell R, Roberts SA, Zubizarreta E, Hendry JH (2012) Dosefractionation sensitivity of prostate cancer deduced from radiotherapy outcomes of 5969 patients in seven international institutional datasets: $\mathrm{a} / ß=1.4$ (09-2.2) Gy. Int J Radiat Oncol Biol Phys 82: e17-e24.

8. Grimm P, Billiet I, Bostwick D, Dicker AP, Frank S, et al. (2012) Comparative analysis of prostate-specific antigen free survival outcomes for patients with low, intermediate and high risk prostate cancer treatment by radical therapy. Results from the Prostate Cancer Results Study Group. BJU Int. 109: 22-29.

9. Thomson D, Merrick S, Swindell R, Coote J, Kelly K, et al. (2012) Doseescalated hypofractionated intensity-modulated radiotherapy in high-risk carcinoma of the prostate: outcome and late toxicity. Prostate Cancer 2012: 450246.

10. Dearnaley D, Syndikus I, Sumo G, Bidmead M, Bloomfield D, et al. (2012) Conventional versus hypofractionated high-dose intensitymodulated radiotherapy for prostate cancer: preliminary safety results from the CHHiP randomised controlled trial. Lancet Oncol 13: 43-54.

11. Albertsen PC, Hanley JA, Barrows GH, Penson DF, Kowalczyk PD, et al. (2005) Prostate cancer and the Will Rogers phenomenon. J Natl Cancer Inst 97: 1248-1253.

12. Hanks GE, Pajak TF, Porter A, Grignon D, Brereton H, et al. (2003) Phase III trial of long-term adjuvant androgen deprivation after neoadjuvant hormonal cytoreduction and radiotherapy in locally advanced carcinoma of the prostate: The Radiation Therapy Oncology Group protocol 92-02. J Clin Oncol 21: 3972-3978.

13. Bolla M, Van Tienhoven G, Warde P, Dubois JB, Mirimanoff RO, et al. (2010) External irradiation with or without long-term androgen suppression for prostate cancer with high metastatic risk: 10-year results of an EORTC randomised study. Lancet Oncol 11: 1066-1073. 